Pneumologe 2010 - 7:315-315

DOI 10.1007/s10405-010-0415-0

Online publiziert: 21. Juli 2010

(c) Springer-Verlag 2010

\author{
R. Loddenkemper \\ Deutsches Zentralkomitee zur Bekämpfung der Tuberkulose (DZK), Berlin
}

\title{
100 Jahre Thorakoskopie
}

Bereits 1937 führte Anton Sattler in

Sehr verehrte Kolleginnen,

Sehr geehrte Kollegen,

am o6. Oktober 1910, also genau vor 100 Jahren, veröffentlichte Hans-Christian Jacobaeus seinen bahnbrechenden Artikel „Über die Möglichkeit, die Zystoskopie bei Untersuchung seröser Höhlungen anzuwenden" in der Münchener Medizinischen Wochenschrift, damals eine der führenden medizinischen Zeitschriften. Gleichzeitig führte er auch die Laparoskopie ein.

Primär hatte Jacobaeus die diagnostische Anwendung der Thorakoskopie im Sinn. So beschreibt er auch in seiner ersten Arbeit zwei Fälle mit tuberkulöser Pleuritis. Dass dies auch heute noch eine gute Indikation sein kann, wird im Beitrag von F. von Groote-Bidlingmaier und C. Bolliger aus Südafrika aufgezeigt.

Wohl als Erster hatte Francis-Richard Cruise in Irland 1866 eine Spiegelung im Pleuraraum durchgeführt. Er nutzte hierfür eine pleurokutane Fistel, die sich bei einem 11-jährigen Mädchen mit einem Pleuraempyem entwickelt hatte. Jacobaeus, der davon nichts wusste, muss aber als der eigentliche Vater der Thorakoskopie gelten, da er die Methode breitflächig bekannt gemacht hat. Schon 1913 führte er die therapeutische Thorakoskopie zur Durchtrennung von Lungenadhärenzen mittels Thorakokaustik ein, um einen kompletten Pneumothorax zu erreichen, eine Methode, die dann für gut 40 Jahre in der Therapie der Lungentuberkulose Anwendung fand (JacobaeusOperation).

Er selbst hat die Thorakoskopie aber u. a. auch beim Pleuraempyem durchgeführt. Auf den heutigen Stand bei dieser Indikation gehen L. Kern und M. Brutsche aus der Schweiz in ihrem Beitrag „Behandlung des Pleuraempyems mittels internistischer Thorakoskopie" ein.
Wien erfolgreich die therapeutische Thorakoskopie beim spontanen Pneumothorax durch, indem er zum Beispiel Adhäsionen durchtrennte, die den Verschluss einer bronchopulmonalen Fistel verhinderten. Heute dient die internistische Thorakoskopie beim Pneumothorax neben der Stadieneinteilung auch dem weiteren therapeutischen Vorgehen, besonders mittels Talkumpuderung. Deren erfolgreiche Anwendung wird von J.-M. Schnyder und J.-M. Tschopp, ebenfalls aus der Schweiz, beschrieben.

Nachdem die Pneumothoraxtherapie der Tuberkulose etwa 1950 mit dem Beginn der spezifischen medikamentösen Behandlung zu Ende ging, wurde die Thorakoskopie auf breiterer Basis bei einer Vielzahl von weiteren Indikationen genutzt. In der bioptischen Diagnostik der diffusen Lungenkrankheiten wurde sie jedoch durch die bronchoalveoläre Lavage und die transbronchiale Lungenbiopsie in Kombination mit der hochauflösenden Computertomografie (HR-CT) abgelöst; bei den lokalisierten Herden der Lunge, der Brustwand und des Zwerchfells durch CT und MRT, und besonders durch die videoassistierte Thoraxchirurgie (VATS), die in den 199oer Jahren eingeführt wurde und sich rasch verbreitete. Heute steht diese chirurgische Thorakoskopie in Konkurrenz zur internistischen Thorakoskopie. Die Unterschiede zwischen den beiden Verfahren arbeiten D. Gompelmann (Heidelberg) und A. Ernst (Boston, USA) heraus, wobei sie auch auf die Unterschiede zwischen internistischer Thorakoskopie mit den starren Instrumenten und dem seit gut 10 Jahren eingeführten semiflexiblen Pleuroskop eingehen.

Die Hauptindikation der internistischen Thorakoskopie liegt heute in der Diagnostik von Pleuraergüssen, die sich auch mittels Pleuraergusspunktion nicht klären lassen. Thorakoskopisch lassen sich so mit hoher Wahrscheinlichkeit maligne oder tuberkulöse Ätiologien ausschließen. Die weiteren Vorteile zeigt W. Frank (Waren, Müritz) in seinem Beitrag auf. Zusätzlich bietet die internistische Thorakoskopie den Vorteil, dass in derselben Sitzung eine Pleurodese mittels Talkuminsufflation (Poudrage) vorgenommen werden kann, die, wie A. Gillissen und S. Kellner (Kassel) schildern, der Goldstandard der internistischen Pleurodesetherapie ist.

Heute stellt die internistische Thorakoskopie/Pleuroskopie ein wichtiges Gebiet innerhalb der interventionellen Pneumologie dar. Diese Ausgabe von Der Pneumologe zur Thorakoskopie kommt passend zum 100-jährigen Jubiläum ihrer Einführung und soll dazu beitragen, dass die Methode, die kostengünstig und relativ leicht zu erlernen ist, vermehrt bei den geschilderten Indikationen zur Anwendung kommt.

Allen Autoren gilt mein Dank für die sorgfältige Erstellung der Beiträge zu diesem Schwerpunktheft.
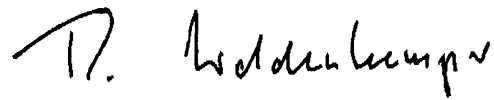

Prof. Dr. R. Loddenkemper

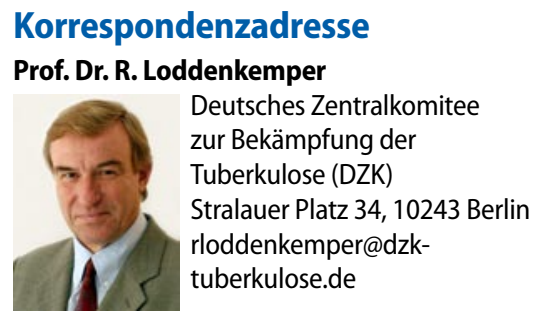

